\title{
Regularities in responding during performance of a complex choice task
}

\author{
Eduardo Mercado III ${ }^{1}$. Vladimir Orduña ${ }^{2}$
}

Published online: 16 June 2015

(C) Psychonomic Society, Inc. 2015

\begin{abstract}
Systematic variations in the rate and temporal patterns of responding under a multiple concurrent-chains schedule were quantified using recurrence metrics and selforganizing maps to assess whether individual rats showed consistent or idiosyncratic patterns. The results indicated that (1) the temporal regularity of response patterns varied as a function of number of training sessions, time on task, magnitude of reinforcement, and reinforcement contingencies; (2) individuals showed heterogeneous, stereotyped patterns of responding, despite similarities in matching behavior; (3) the specific trajectories of behavioral variation shown by individuals were less evident in group-level analyses; and (4) reinforcement contingencies within terminal links strongly modulated response patterns within initial links. Temporal regularity in responding was most evident for responses that led to minimally delayed reinforcers of larger magnitude. Models of response production and selection that take into account the time between individual responses, probabilities of transitions between response options, periodicity within response sequences, and individual differences in response dynamics can clarify the mechanisms that drive behavioral adjustments during operant conditioning.
\end{abstract}

Keywords Behavior dynamics · Individual variations · Matching law $\cdot$ Neural networks $\cdot$ Reinforcement schedules

Eduardo Mercado, III

emiii@buffalo.edu

1 Department of Psychology, Park Hall, University at Buffalo, The State University of New York, Buffalo, NY 14260, USA

2 Facultad de Psicología, Universidad Nacional Autónoma de México, Mexico City, Mexico
A basic assumption of several theories of operant conditioning is that group-level measures of performance are sufficient for understanding the mechanisms of learning within individuals (Pear, 2001; Rachlin, 2000; Staddon \& Cerutti, 2003; Williams, 1990). Although there is good evidence that this is often not the case (Gallistel, Fairhurst, \& Balsam, 2004; Gallistel et al., 2007), what kinds of behavioral descriptions might best address such complications is unknown. Possibly, modeling behavior at the level of the individual would suffice. But, if the mechanisms engaged during learning vary significantly across individuals, then fitting a single uniform learning model to individual performances might be just as inappropriate as assuming that group-level models adequately describe behavior (e.g., see Worthy, Hawthorne, \& Otto, 2013). Gaining a clearer understanding of which aspects of learned behavior are prevalent within and across individuals, and the extent to which those properties are predictable, is thus critical to choosing measures of behavior that most clearly reveal the nature of the mechanisms that drive long-term changes in behavior. The purpose of the present study was to quantitatively assess the regularity ${ }^{1}$ of responses produced by individual subjects performing in a relatively complex operant conditioning task to determine which measures of behavior best characterized changes in subjects' response patterns as a function of training conditions.

Early in the history of animal learning research, it was discovered that training animals within constrained environments often led subjects to perform stereotyped actions. For instance, Guthrie and Horton (1946) collected photographic

\footnotetext{
${ }^{1}$ In the following, the term regularity is used to refer to patterning in the timing, sequencing, or form of responses. Stereotypy is used to specify regularity in the form of movements, and periodicity is used to indicate that responses occur at regular/rhythmic intervals.
} 
records of cats escaping from puzzle boxes which showed that, after conditioning, each cat's body position was highly similar at the moment the cat activated the door-opening sensor from one trial to the next. Their study also revealed, however, that cats' escape postures were less stereotyped when (1) the position or reliability of the sensor varied across trials, (2) subjects' movements while entering the box were variable, and (3) subjects were more excitable. Furthermore, the stereotyped postures that cats adopted varied considerably across cats, with each cat appearing to develop its own idiosyncratic means of activating the door sensor. Thus, cats were shown to both consistently reproduce individual-specific complex actions when faced with highly similar circumstances and to perform a wide variety of different actions when either internal or external factors varied slightly.

Subsequent studies examining the predictability of behavior in different experimental contexts have focused less on the particulars of movement patterns of individuals and more on global patterns of response production associated with different schedules of reinforcement (Baum, 2002; Baum \& Rachlin, 1969; Herrnstein, 1970; Nevin \& Grace, 2000). Such molar measures of responding provide the foundation for many modern theories of operant conditioning and have been particularly important in clarifying how animals process temporal cues and make response decisions during conditioning (for review, see Staddon \& Cerutti, 2003). A minority of researchers has attempted to supplement this approach with more molecular measures of responding during learning (Blough, 1963; Fantino \& Royalty, 1987; Newland, 1997; Palya, 1992; Shimp, 1992; Silva \& Pear, 1995; Silva, Pear, Tait, \& Forest, 1996; Weiss, 1970), and to use such measures to develop alternative theories of conditioning (Galbicka, 1992; Misak \& Cleaveland, 2011; Shimp, 1966; Shimp, Fremouw, Ingebritsen, \& Long, 1994; Tanno \& Silberberg, 2012). For instance, Silva and Pear (1995) analyzed measures of pigeon's spatial trajectories as the birds learned to respond to fixed-time (FT) and fixed-interval (FI) reinforcement schedules and found that movement stereotypy varied as a function of when rewards were delivered and on whether rewards were contingent on pecking. Palya (1992) analyzed the timing of pecks by pigeons performing in hundreds of consecutive sessions with different reinforcement schedules and showed that pigeons consistently pecked at a rate of $\sim 3 \mathrm{~Hz}$ regardless of schedule. Other features of interresponse time (IRT) distributions, however, were idiosyncratic to particular birds. Pear (1985) similarly reported that pigeons performed elaborate, idiosyncratic, periodic movement patterns when pecking in response to a variable interval schedule. Thus, like cats escaping from puzzle boxes, pigeons pecking for seeds produce a combination of both consistently recurrent actions and idiosyncratic response patterns. These types of detailed analyses of responding during conditioning have established that animals not only reproduce similar actions in similar contexts, but also that the temporal dynamics of those responses can be quite stereotyped and individualspecific.

Past studies of movement regularity during conditioning have described what animals are doing as they acquire or perform responses under basic reinforcement schedules (Blough, 1963; Guthrie \& Horton, 1946; Li \& Huston, 2002; Li, Krauth, \& Huston, 2006; Palya, 1992; Silva \& Pear, 1995; Silva et al., 1996). Unsurprisingly, subjects presented with such schedules show greater consistency in response timing when the timing of reinforcement is more predictable (e.g., during FI and FT schedules). Even with such simple schedules, however, detecting temporal regularity in response patterns may require analyzing time windows that include multiple responses ( $\mathrm{Li} \&$ Huston, 2002; Li et al., 2006), or preclassifying extended bouts of responding (Kirkpatrick \& Church, 2003; Shull, Gaynor, \& Grimes, 2001). Consequently, the extent to which spatiotemporally regular responding observed during conditioning tasks is a consequence of periodicities within the schedules of rewards that animals are experiencing versus the result of predispositions to produce predictably timed response patterns in contexts that do not explicitly promote them remains unclear. In the current study, we attempted to overcome these limitations by using nonlinear signal processing algorithms and neural networks to analyze the regularity of responses produced by rats at multiple time scales, in a more complex operant task involving multiple response options, and no explicit reinforcement of timed responses.

\section{Analyzing temporal regularities in response patterns}

The specific analysis techniques applied in the current study were selected to take advantage of existing metrics for describing recurrent sequences developed in other fields, while making use of response measures that are readily obtained using standard operant conditioning technologies. Multiple overlapping measures of response regularity, assessed at multiple time scales and across multiple response options, were included in the analysis to reduce the likelihood of metricspecific artifacts; several of these measures have never previously been applied to behavioral sequences. Fourier analyses of response patterns, which have been used successfully in past studies of behavioral stereotypy/periodicity within operant conditioning tasks (Silva \& Pear, 1995; Silva et al., 1996), were not used in the current study because preliminary analyses suggested that the key periodic features of response sequences were more simply and adequately characterized using alternative measures of recurrence. Temporal regularity in responding can arise from intrinsic tendencies to produce rhythmic movements, cyclical movements between response options, and the performance of highly stereotyped movement patterns during responding. 
A standard approach to quantifying temporal regularities in responses within operant conditioning tasks is to create histograms of IRTs (Gentry, Weiss, \& Laties, 1983; Iversen, 1991; Palya, 1992). Variations in the qualities of IRTs across different reinforcement schedules provide summaries of the variability of response timing similar to those revealed by cumulative records of responses. Measures of IRTs can be easily extracted from records of response times, and the shape of the histogram created from these measures can often be adequately described using simple mathematical functions, such as the exponential function (e.g., Sidman, 1954), Markov chains (Cleaveland, 1999), or the log survivor plot (Shull et al., 2001). Additional measures are required to characterize sequential temporal structure across IRTs. One approach that has often been used in quantitative analyses of behavior is to create recurrence or return plots (Webber \& Zbilut, 1994), by plotting consecutive IRTs against one another (Eckmann, Oliffson Kamphorst, \& Ruelle, 1987; Li \& Huston, 2002; Marr, 1992; Palya, 1992). Such graphs can visually reveal characteristic periodic features within response sequences (Li et al., 2006), but detailed features of the graphs are sometimes difficult to summarize quantitatively (Webber \& Zbilut, 1994)

In the current study, an alternative measure of recurrence called the recurrence period density entropy (RPDE) was used to quantify temporal regularities in behavioral response patterns. The RPDE measure originally was developed for quantifying variations in the periodicity of biological signals such as electrocardiograms and speech recordings (Little, McSharry, Moroz, \& Roberts, 2006; Little, McSharry, Roberts, Costello, \& Moroz, 2007). This measure takes on a value between zero (for purely periodic signals) and one (for random signals, like white noise). The value is calculated by converting a time series $x_{n}$ comprised of $n$ measurements sampled at equal intervals into a matrix representing multiple time-delayed versions of that time series:

$\mathrm{X}_{n}=\left[x_{n}, x_{n+\tau}, x_{n+2 \tau}, \ldots, x_{n+(\mathrm{M}-1) \tau},\right]$,

where $\tau$ is the delay and $M$ is the number of dimensions included within the matrix. Once this matrix has been created, the number of times the signal returns within a fixed distance of each point (defined as a vector) within this M-dimensional space is counted, and the periods between successive returns are cumulated as a histogram (see Fig. 1). This histogram is normalized to sum to one to create a recurrence period density function $P(T)$, where $T$ is the time between returns. The normalized entropy of this function:

$H_{\text {norm }}=-\left(\ln T_{\text {max }}\right)^{-1} \sum_{t=1}^{T_{\max }} P(t) \ln P(t)$

provides the RPDE value, where $T_{\max }$ is the largest recurrence period. In addition to RPDE measures, periodic peaks in autocorrelation functions were used as a secondary measure of temporal regularity. Although RPDE measures provide a way to quantify behavioral variability, and in particular the periodicity of responding, they do not directly reveal the specific patterns of response timing that rats produce. Other behavioral measures, such as IRTs, more directly indicate the periods at which rats repeat responses.

These measures of temporal recurrence/periodicity were applied to the raw time series data of individual responses, to automatically segregated "bouts" of identical responses, and to constructed waveforms representing the time series of transitions (switches) between different response options. Transitional probabilities between response options were also calculated to provide an assessment of the stability of timeindependent sequential structure within response patterns. Finally, traditional measures of response rate were calculated to facilitate direct comparisons with recurrence metrics and transitional probabilities. The goal of this approach was to provide a broad, objective assay of each subject's behavior during performance of the operant conditioning task, to thereby increase the likelihood that systematic changes in response regularity associated with learning would be detectable.

Measures of all recorded responses made by subjects were included in the analysis, making it possible to examine not only response patterns within subcomponents of a single session, but also variations across sessions. Figure 2 summarizes the temporal measures of response regularity included in the current analysis. By analyzing the temporal regularity of responses produced by individuals within an operant chamber, one can not only detect any patterns in the timing or sequencing of responses produced by those individuals, but can also assess the stability of such patterns over time, across individuals, and in varying conditions. Such measures can also be used to evaluate whether the timing of individual responses or bouts of responses is probabilistic (as assumed by several current theories of responding in choice tasks) or more predictably patterned, as well as whether regularity in responding (or lack thereof) is uniform across individuals.

\section{Sorting behavioral response patterns with self-organizing maps}

Traditional summaries of behavioral descriptions have relied heavily on categorical descriptors, data plots, linear regression models, and sample statistics. Such analyses clearly provide useful information about behavioral regularities and can be particularly useful when the goal is to use inferential statistics to test a specific hypothesis. These approaches are more limited, however, when it comes to characterizing complex variations in response patterns that may be task- or individualspecific (Mercado, 2011). When the goal is to describe such 


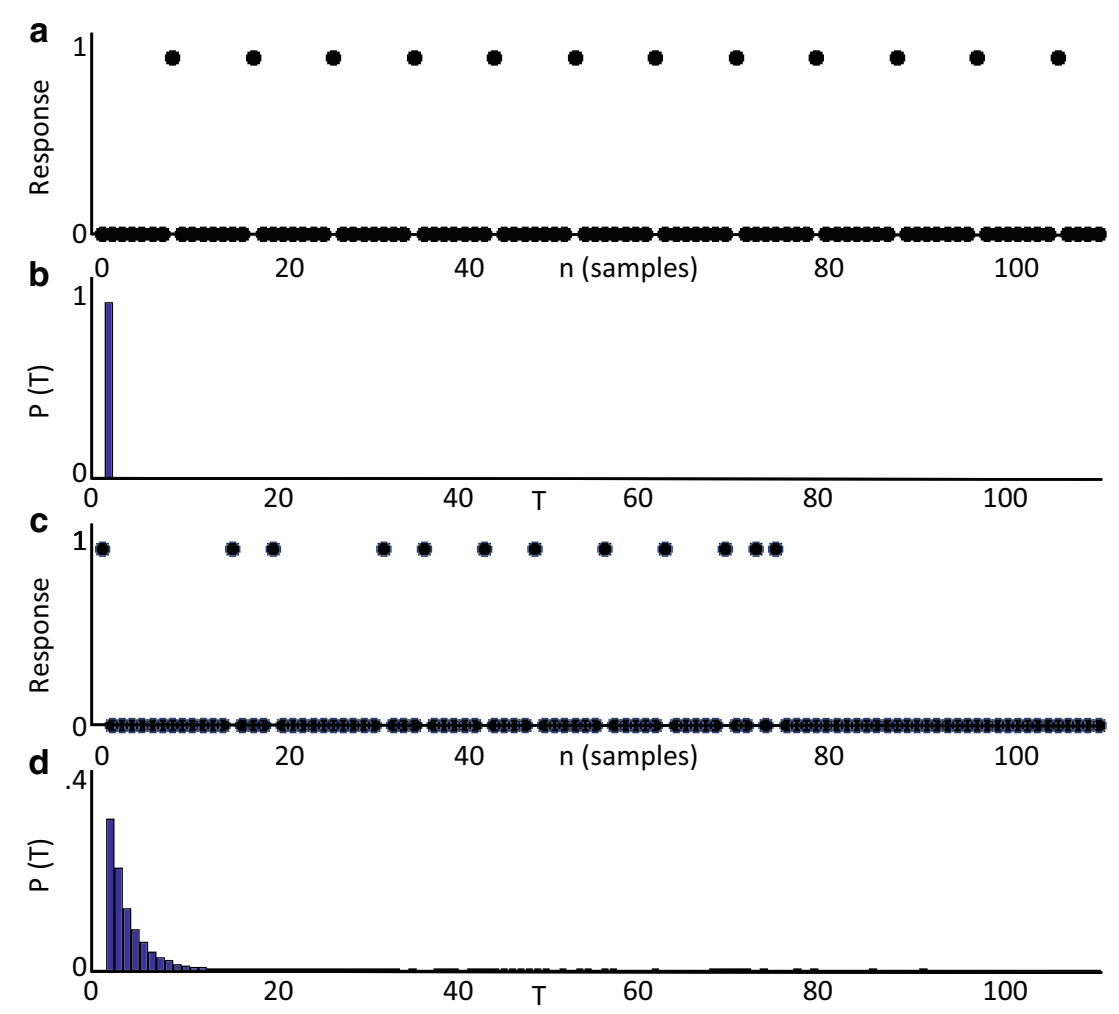

Fig. 1 (a) Idealized periodic lever presses (shown as circles; response value $=1$ ) occur at fixed intervals. (b) The recurrence period density function $P(T)$ for this periodic response pattern shows that almost all repetitions occur at the same interval $T$, giving an entropy value close to zero. (c) Randomly ordering the time series data from (a) produces a
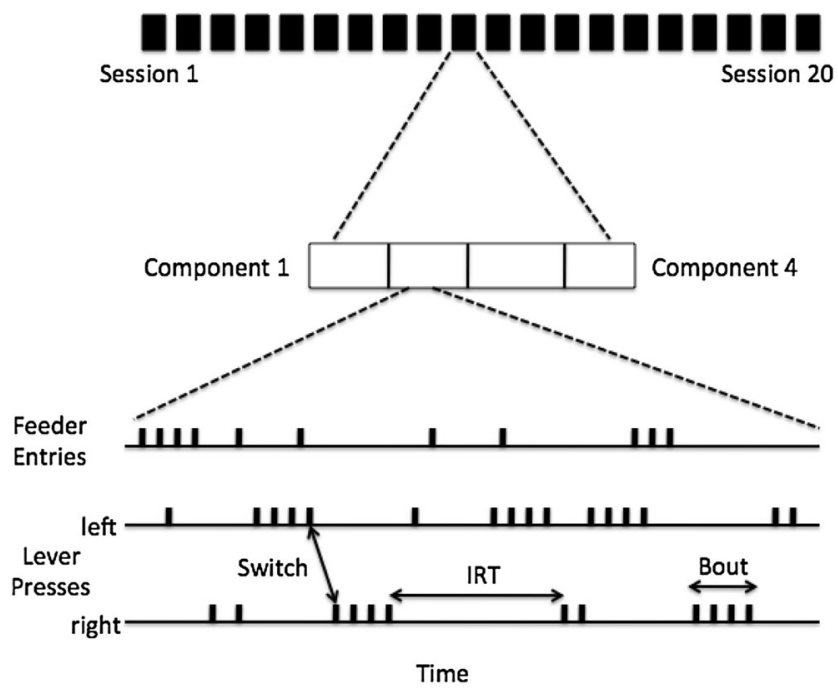

Fig. 2 Measures of temporal regularity in the current study included interresponse times (IRTs) for each of three response options (presses to two levers, and feeder entries), rate of responding, number and durations of response bouts and switches between response options, and measures of periodicity within the time series of individual responses as well as time series of response bouts. These measures were collected for data from four distinct training components within each session; alternating components featured distinctive reinforcement contingencies. Data from 20 consecutive sessions were analyzed, including the first sessions that rats were exposed to the behavioral task much less periodic time series (only the initial samples in the full time series containing $\sim 10,000$ samples are shown). (d) The recurrence period density function $P(T)$ for this randomly permuted time series shows that responses repeat at multiple intervals; the entropy of this function is thus greater than for the more periodic response pattern

variations and to generate new hypotheses, exploratory analyses such as factor analysis, principal components analysis, and multidimensional scaling are often more useful (Budaev, 2010; Ossenkopp, Sorenson, \& Mazmanian, 1994). A basic assumption of traditional approaches to describing behavioral variation with exploratory analysis is that the dimensions along which behavior is varying are stable and uniform across individuals. Consequently, these methods are often inadequate for describing behavior that changes over time and that differs significantly across individuals (Mercado, 2011).

An alternative approach to characterizing behavioral variations is to automatically sort descriptors of behavioral patterns based on their similarity and frequency of occurrence. Self-organizing neural networks have been widely used for this purpose in other fields (e.g., Lloyd, Wongravee, Silwood, Grootveld, \& Brereton, 2009). In the current study, a neural network known as the self-organizing map (Kohonen, 2001, 2013) was used to automatically sort sets of behavioral descriptors. Self-organizing maps (SOMs) are often used to visualize data in ways that facilitate the identification of clusters of similar patterns as well as the relationships between key features that differentiate those patterns. SOM analyses are similar to multidimensional scaling in that both approaches sort inputs based on their similarity. However, unlike 
multidimensional scaling, SOMs develop a fixed number of "prototypes" that correspond to frequently encountered inputs, and spatially organize these prototypes in two dimensions such that adjacent prototypes within an SOM are more similar than nonadjacent ones. This feature of SOMs can sometimes reveal clusters that are less apparent in multidimensional scaling analyses (Kirt \& Vainik, 2007).

In the current study, SOMS were analyzed to determine which patterns of responding to levers and a feeder varied systematically within and across individuals, sessions, and conditions, as well as how those patterns changed with training. SOM analyses also made it possible to objectively identify prototypical and idiosyncratic patterns of responding by individuals. The basic goal of automatically sorting behavioral response patterns in the operant conditioning task was to determine the extent to which there were predictable regularities in individual rat's patterns of responding and, if so, how those patterns varied over time.

SOMs consist of an $M \times N$ grid of computational nodes (see Fig. 3a) that initially respond selectively to random inputs. Each input to an SOM consists of a vector $\left(\mathbf{y}_{i}\right)$. Nodes in

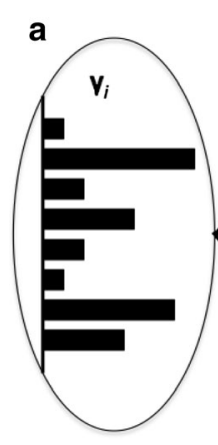

b

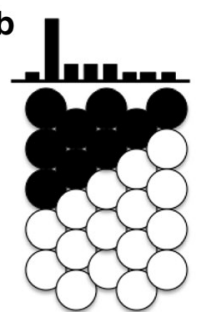

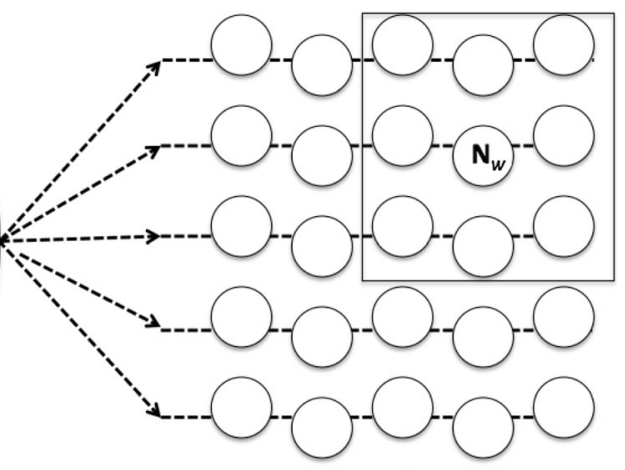

C

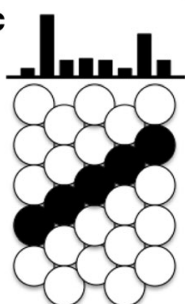

d

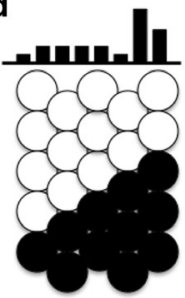

Fig. 3 (a) An input vector $\mathbf{y}_{i}$, each element of which corresponds to a quantified measure of response patterning, is compared to each node within a self-organizing map (SOM) to determine which node is most similar $\left(\mathbf{N}_{w}\right)$. Nodes surrounding the winner node (inside the square) will generally respond well to similar inputs after training. When trained with a set of systematically varying inputs, the spatial organization of an SOM reflects the structure of the inputs. (b) Black nodes respond well to inputs in which the second element is high and all others are low; these nodes are clustered in the opposite corner from nodes that respond well to inputs in which elements 7 and 8 are high, but element 2 is low (shown in d). (c) Nodes in the middle of the map respond best to inputs with qualities between these two extremes. The diagonal structure of node sensitivities is a consequence of variations within the input patterns used to train the SOM. In this way, the SOM spatially sorts inputs (in this case response patterns) based on their similarities and differences the map are also represented as vectors $\left(\mathbf{n}_{i}\right)$. The full map is represented by a matrix. Nodes in the map compete whenever an input is presented and the node that is most similar to $\mathbf{y}_{i}$ wins the competition. Input connections to the winning node, as well as nodes surrounding that node are then adjusted using a learning algorithm, such as the following, so that the nodes will respond more strongly when that input is encountered again:

$\mathbf{n}_{i}(t+1)=\mathbf{n}_{i}(t)+g_{w i}(t)\left[\mathbf{y}_{i}(t)-\mathbf{n}_{i}(t)\right]$,

where $g_{w i}(t)$ is a function, called the neighborhood function, that determines which nodes are modified and to what extent. For instance, if the neighborhood function $g_{w i}(t)$ is specified as:

$g_{w i}(t)=\alpha(t) \exp \left[-\operatorname{sqdist}(c, i) / 2 \sigma^{2}(t)\right]$,

where $\alpha(t)$ is a decreasing function of $t, \operatorname{sqdist}(c, i)$ is the squared distance between nodes $c$ and $i$, and $\sigma(t)$ is a second decreasing function of $t$, then the number of nodes surrounding the best matching node that are modified during learning will gradually decrease as map training progresses. Repeated presentations of input sets to an SOM can cause the sensitivities of its nodes to become spatially organized such that dimensions along which inputs vary correspond to spatial dimensions within the map (see Fig. 3.b-d).

SOMs can be trained using inputs created from measures of an individual's behavior, or using measures from multiple individuals. The structure of maps can thus be used to summarize how response patterns vary both within and across individuals. For instance, one can tally the number of times each SOM node is activated by an input set to identify which response patterns are most typical (see Fig. 4a). By analyzing which inputs activate each node, it is also possible to determine whether certain conditions lead to systematic variations in response patterns (see Fig. 4b). Node vectors can be interpreted as weights that are assigned to each element of an input vector. By examining the relative weights given to each behavioral measure by each node (referred to as component planes), it is possible to determine which measures in particular led to the spatial organization of an SOM (see Fig. 4c), as well as how consistently specific measures vary as a function of different experimental conditions (compare Fig. $4 b$ to $c)$.

Often, visual inspection of SOM properties can reveal how it is sorting input patterns. However, it is also possible to quantitatively analyze the structure of an SOM by describing it using a hit distribution vector (see Fig. 4a), in which each element value corresponds to the number of times each node was activated by an input. For instance, if all inputs of one type, such as response measures collected early in training, are presented to an SOM to create one hit distribution vector, and 

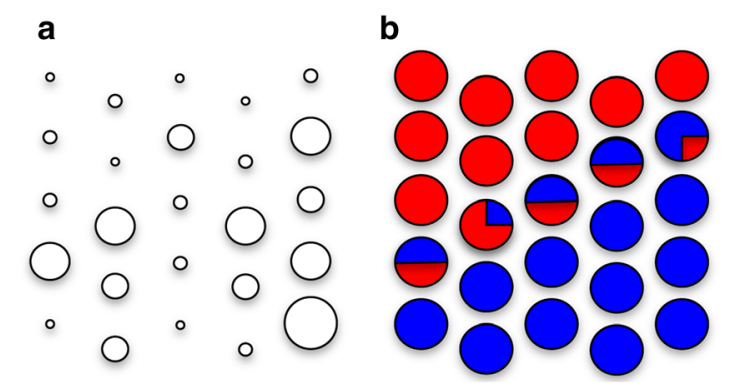

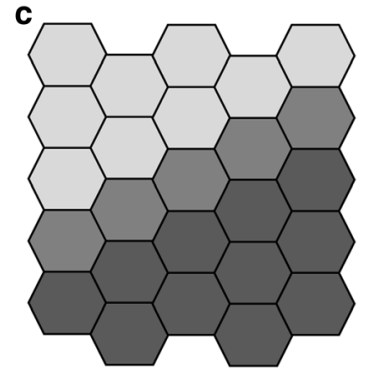

Fig. 4 (a) The number of times each node within a self-organizing map (SOM) best matches an input can be depicted by varying the sizes of circles representing nodes; larger circles = more "hits." In this example, inputs similar to the pattern shown in Fig. $3 \mathrm{~d}$ are prevalent. Tallies can be used to create a hit distribution vector (here, a 25 -element vector) to facilitate quantitative comparisons of SOMs. (b) If inputs can be categorized (e.g., early training sessions $=$ red/light gray; later sessions $=$ blue/dark gray), then the proportion of inputs from each category that best matches each node can be calculated; solid colors indicate that $100 \%$ of inputs were from the same category. (c) Component planes show the sensitivity of each node to elements of input vectors. The component plane shown here for the seventh element of the input vectors (see Fig. 3b-d) shows that nodes in the lower right corner respond best when this measure is higher (darker shading), while nodes in the upper left corner respond when it is lower (lighter shading); the spatial organization of this SOM reveals systematic differences between early and late responding all inputs of a second type (e.g., measures collected late in training) are used to create a second hit distribution vector, then the difference between these two vectors provides an index of how selectively SOM nodes responded to inputs of each type. If most nodes respond selectively to inputs of one class, then the difference will be large. For instance, in the example shown in Fig. 4, the SOM shows high spatial structuring, such that the hit distribution vectors for early versus later responses have little overlap. These same calculations can be made for each component plane of an SOM, making it possible to rank each input element (behavioral measure) according to how strongly it affected the spatial organization of an SOM (Lloyd et al., 2009). Because behavioral measures will vary in range, it is first necessary to normalize each component plane to values between zero and one across all map units. Each plane can then be used to scale the hit distribution vector for each input category. A ratio $z$ is calculated to quantify how closely variations in each element map onto category-related variations in map structure:

${ }^{\mathrm{aj}} z=\sum_{m=1}^{M} \sum_{n=1}^{N}{ }^{\mathrm{a}} r_{m n}{ }^{j} v_{m n} / \sum_{m=1}^{M} \sum_{n=1}^{N}{ }^{\mathrm{a}} r_{m n}$,

where $a$ is the number of categories, $j$ is the number of elements in the input vector, $M$ and $N$ are the SOM dimensions, $r$ is each normalized hit distribution vector value, and $v$ is each normalized component plane value. The higher the ratio (called the SOM Discrimination Index), the closer the behavioral measure corresponds to the category-related structure in the SOM. For example, in Fig. 4, the categorical structure of the SOM (Fig. 4b) is highly similar to the component plane for the seventh element of the input vector (see Fig. 4c), which would give a high $z$ value for that behavioral measure. Other behavioral measures that do not systematically vary across the
SOM, such as the first element of the input vector shown in Fig. 3b-d, give lower $z$ values.

One advantage of using SOMs as a pattern sorting technique is that it is not necessary to prespecify the categories or dimensions of variation along which inputs are to be classified. When several behavioral measures are presented as inputs to an SOM, the structure of the trained map will reflect both the frequency with which similar sets of descriptions are presented to the map as well as the consistency with which the descriptors vary. If a measure never varies across inputs (e.g., if response rates were stable for all conditions over time), then that measure will have little impact on the spatially organized sensitivity of the map, because all of the map nodes will learn to respond approximately equally to that measure. If a behavioral measure varies unsystematically, it will also have little effect on the spatial organization of the SOM, because the specific values presented will be relatively infrequent and will lead to counteracting changes in node sensitivities. In contrast, behavioral descriptors that vary systematically along a continuum will strongly impact the spatial organization of the SOM, such that nodes positioned at opposite locations within the map will be most sensitive to values at opposite ends of the continuum of values within the input set (as illustrated in Fig. 3b-d). As a result, SOM analyses can link response pattern variations to experimental conditions in ways that would be difficult to replicate with traditional statistical approaches.

\section{Behavioral analysis}

In the current study, the structure of SOMs trained with a combination of molar and molecular measures of behavioral responding in an operant chamber were compared within and across individuals as a function of time spent within a training session, days of training, variations in reward magnitude, and variations in reinforcement schedules. These comparisons 
provided a way to objectively assess whether behavioral patterns produced by rats in a relatively complex choice task varied systematically in terms of both temporal regularity and more traditional measures of response rate. Based on past studies showing response stereotypy as well as idiosyncratic response patterns of individuals performing operant tasks, we expected that rats would show variable behavior early in training but more regular responding in later training sessions. We also expected that asymptotic patterns of responding might be heterogeneous across individuals.

The behavioral data analyzed here were collected by Orduña and colleagues (2013). The original experiment was designed to examine the effect of magnitude of reinforcement on the sensitivity to delay within a two-component concurrent-chains schedule (described below). In the current analysis, the degree of regularity in lever pressing and nose poking actions of rats was quantified by analyzing the time series of responses produced during training, as well as more molar measures of response rate. Details of the subjects and experimental procedure are provided by Orduña et al. (2013), and will only be briefly summarized here.

\section{Subjects}

The subjects were 12 experimentally naïve male Wistar rats, approximately 90 days old. Subjects were food restricted and maintained at approximately $85 \%$ of their free feeding weight. Water was available ad lib in the home cage.

\section{Apparatus}

Six operant conditioning chambers (MED Associates, Inc., Model ENV 008-VP) served as the experimental spaces. Each chamber had two retractable response levers (MED Associates, Inc., Model ENV-112CM) located $10.5 \mathrm{~cm}$. above the floor, in the front wall. Each lever was $4.8 \mathrm{~cm}$. wide. The visual stimuli used were two triple stimulus displays, each of them situated $1.5 \mathrm{~cm}$ above each lever. Each triple stimulus display consisted of a bar of acrylic mounted on an aluminum bar with three apertures $1 \mathrm{~cm}$ in diameter and separated by $.6 \mathrm{~cm}$, capable of projecting (from left to right) red, white, and blue light via ultrabrillant LEDs. A $5.1 \mathrm{~cm} \times 5.1 \mathrm{~cm}$ pellet receptacle (MED Associates, Inc., Model ENV-200R2M) was located in the center of the front wall, $2.5 \mathrm{~cm}$ above the floor. The receptacle received, according to the schedule, $45 \mathrm{mg}$ Noyes precision food pellets (Bio-Serv, Product F0165) from a circular modular pellet dispenser (MED Associates, Inc., Model ENV-203 M). Entries to the feeder were measured with a head-entry detector (ENV-254-CB). The presentation of stimuli and the collection of data were controlled by personal computers using the Medstate programming language (MedPC-IV, MED Associates, Inc.).

\section{General experimental procedure}

Rats were first trained to press the lever employing an alternative schedule FT $45 \mathrm{~s}$ fixed-ratio 1 . When subjects obtained 80 reinforcers in a $30 \mathrm{~min}$ session, they were switched to a random-interval $15 \mathrm{~s}$, in the left and right levers, in alternating sessions, until they obtained 50 reinforcers for two consecutive sessions in each lever; after those sessions, the randominterval schedule was changed to $30 \mathrm{~s}$ until the same criteria were achieved. After this pretraining, subjects were evaluated in a two-components multiple concurrent-chains schedule, signalled by different stimuli in the triple stimulus display mounted over each lever. In both components, the initial links were nonindependent variable interval $15 \mathrm{~s}$, and the terminal links were associated to a different delay to reinforcement (FT schedules for half of the subjects, FI schedules for the other half) - for example, $2 \mathrm{~s}$ in the left lever, $28 \mathrm{~s}$ in the right lever. The differences between components were (1) the location of the short delay alternative, and (2) the magnitude of the reinforcer - one pellet for each alternative in one component and four pellets for each alternative in the other component.

Nonindependent VI schedules with a $2 \mathrm{~s}$ changeover delay were employed in the initial links for each component, while FT or FI (according to the group) were presented in the terminal links. The important difference between the terminal links within a component was the delay to reinforcement. Across conditions, the following pairs of delays were employed (228 ; $6-24 ; 24-6$; $28-2 \mathrm{~s}$, for left and right alternatives, in conditions $1-4$, respectively). When 12 reinforcers had been obtained in this component (red/left), there was a $2 \mathrm{~min}$ intercomponent interval, after which the other component was initiated. In this new component there were different discriminative stimuli, but the same pairs of delays were employed (28-2; 24-6; 6-24; $2-28 \mathrm{~s}$ in conditions $1-4$, respectively). Note that the short delay alternative position was changed between components. The most important difference between components was the magnitude of reinforcer. This cycle was repeated twice, such that there were 4 components in each session. Each condition was presented for 20 sessions; the conditions were conducted in different orders across subjects.

\section{Quantitative analyses}

Orduña et al. (2013) plotted and statistically analyzed rats' response rates in the initial links during the final five sessions of training as a function of different reinforcer magnitudes and terminal link delay conditions, whereas in the present study the regularity of response patterns across all training sessions was quantified and analyzed with SOMs. Furthermore, systematic variations in the responses of individual rats were analyzed and compared to those evident at the group level. To simplify the analyses, only responses from Condition $1(2 \mathrm{~s}$ 
vs. $28 \mathrm{~s}$ delay in the terminal link) were included in the current study.

As noted above, rats alternated between components in which their responses led to either smaller (one pellet) or larger (four pellets) rewards, such that sessions were divided into four components. All behavioral measures described below were collected for individual components, providing four samples of responding per session.

Measuring response patterns Behavior within concurrent reinforcement schedule tasks is commonly viewed as indicative of a subject's choices when faced with multiple, potentially differentially rewarding options. In the current task, recorded response choices included pressing the left lever, pressing the right lever, or visiting the feeder; each choice was potentially rewarding. We measured the response rates to levers during the initial links in the concurrent chains, as well as across both initial and terminal links. We also measured the rate of feeder entries, the transitional probabilities of switching between levers, the number of times rats switched between levers, and the transitional probabilities of returning to a lever after visiting the feeder.

Customized Matlab scripts were created to automatically extract measurements of temporal response patterns from MedAssociates data recorded during training sessions. IRTs were measured for left and right lever presses, as well as for feeder entries, and then summarized with histograms (bin size $=.5 \mathrm{~s}$ ). We also created a histogram from the IRTs of all three response options interleaved to provide a global measure of response timing. Curve fitting functions within Matlab were then used to fit an exponential function to each IRT histogram; the two parameters $(a, b)$ determining the shape of the function $a^{*} \exp \left(b^{*} x\right)$ served as metrics summarizing the distributions of IRTs for each response option. Because the lever associated with short ( $2 \mathrm{~s}$ ) or long (28 s) delay rewards alternated between components within a session, the current analysis focused on IRTs associated with whichever lever led to the short delay (hereafter referred to as the "short-delay" lever) or to a long delay (the "long-delay" lever) rather than on the left or right position of levers. MedAssociates data records were also used to generate time series data for lever presses and feeder entries. Recurrence period density entropies (RPDEs) were measured from these time series vectors using the $r p d e()$ Matlab function developed by Little and colleagues (Little et al., 2007).

Repetitive selection of a particular choice action can potentially be viewed as a response bout (Blough, 1963; Kirkpatrick \& Church, 2003; Shull et al., 2001). In tasks where only one response option is available, bouts can be identified based on longer IRTs that separate them. However, it is often difficult to objectively segregate response bouts (Shull et al., 2001). In the current analysis, bouts were operationally defined as starting or ending whenever a rat performed a response that differed from its previous response (see Fig. 1). From time series of bouts, we measured the number, median duration, and coefficient of variation of the duration of bouts for responses to each lever. The relatively small number of bouts in some sessions precluded use of RPDE measures for quantifying the periodicity of bouts to each lever, so an alternative measure of periodicity was created by comparing the autocorrelation function of a series of bout durations to the autocorrelation function of a sorted series of the same durations. The sum of the absolute difference between these functions produced values that were higher for more periodic bout series. Finally, the regularity of bouts of all three response options combined was quantified by calculating the RPDE of the time series for all three response options, which was created by assigning different values to different response options over time (short-delay response lever $=1$; feeder entry $=0$; long-delay response lever $=-1$ ).

Table 1 summarizes the 28 measures of behavior collected from rats performing in the two-components multiple concurrent-chains schedule. The main goals of collecting this broad range of measures were to provide a more complete description of the dynamics of rats' actions over time than is typically considered in operant conditioning experiments, and to assess the regularity of responses at multiple time scales.

Analyzing variations in response patterns The various behavioral measures described above were combined to create sets of 28-element vectors describing the behavior of rats within single components of sessions. Because there were four components per session, and 20 sessions per rat, the behavior of each rat within and across sessions could be described with an $80 \times 28$ matrix of behavioral measures. Matrices of behavioral descriptors were used to train SOMs.

SOMs were trained and analyzed in two ways. First, the freely available data mining program Orange (Demsar, Zupan, Leban, \& Curk, 2004) was used to create a single, highresolution $6 \times 6$ map for each rat. This software allows for data analysis using drag-and-drop modules, and thus allows SOMs to be easily implemented by nonprogrammers. The choice of a 36 node SOM was made through trial and error to minimize the number of nodes activated by few or no input patterns (Kohonen, 2013). A hexagonal map architecture was selected to increase the number of nodes surrounding each node in the SOM, so that finer gradations in spatial structure could be resolved. Default neighborhood functions and learning rate parameters set by the Orange program were used for training all SOMs.

After each SOM was trained, component planes for each of the 28 behavioral measures were visually inspected to determine which measures contributed to the spatial organization of the map. Component planes were subjectively classified based on whether they showed clear spatial organization, some evidence of spatial organization, or no obvious spatial 
Table 1 Twenty-eight behavioral measures collected from rats performing in the two-components multiple concurrent-chains schedule

\begin{tabular}{|c|c|}
\hline \multicolumn{2}{|l|}{ Behavioral Measures } \\
\hline $\begin{array}{l}\text { Response rate } \\
\text { Initial link -2 } \\
\text { Initial link -28 } \\
\text { Initial link + TL -2 } \\
\text { Initial link + TL -28 } \\
\text { Feeder entry }\end{array}$ & $\begin{array}{l}\text { Response rates were calculated by dividing } \\
\text { the number of responses by the duration } \\
\text { over which responses were collected }\end{array}$ \\
\hline $\begin{array}{c}\text { Response intervals } \\
\text { Initial link -2 }(a) \\
\text { Initial link -2 }(b) \\
\text { Initial link -28 }(a) \\
\text { Initial link -28 }(b) \\
\text { Feeder entry }(a) \\
\text { Feeder entry }(b) \\
\text { All responses }(a) \\
\text { All responses }(b)\end{array}$ & $\begin{array}{l}\text { Exponential functions were fit to histograms } \\
\text { of measured interresponse intervals } \\
\text { (bin size }=.5 \mathrm{~s} \text { ); } a \text { and } b \text { parameters for } \\
\text { each exponential fit to a particular } \\
\text { histogram were used as descriptors for } \\
\text { that histogram }\end{array}$ \\
\hline Transitions & \\
\hline $\begin{array}{l}\text { Number of switches } \\
\text { Switch bias } \\
\text { Return bias }\end{array}$ & $\begin{array}{l}\text { All transitions between levers were counted } \\
\text { as switches }\end{array}$ \\
\hline Bout properties & \\
\hline $\begin{array}{l}\text { Number of bouts - } 2 \\
\text { Number of bouts }-28 \\
\text { Median bout DUR -2 } \\
\text { Median bout DUR -28 } \\
\text { CV bout DUR -2 } \\
\text { CV bout DUR - } 28\end{array}$ & $\begin{array}{l}\text { Bouts were defined by transitions between } \\
\text { response options and were analyzed } \\
\text { separately for each lever }\end{array}$ \\
\hline Response periodicity & \\
\hline $\begin{array}{l}\text { RPDE Initial link -2 } \\
\text { RPDE Initial link -28 } \\
\text { RPDE Feeder entries } \\
\text { RPDE response bouts } \\
\text { ACD bouts }-2 \\
\text { ACD bouts }-28\end{array}$ & $\begin{array}{l}\text { Recurrent features of response and bout } \\
\text { sequences were calculated for the entire } \\
\text { time series of responses/bouts within } \\
\text { each component }\end{array}$ \\
\hline
\end{tabular}

Note: Italicized measures correspond to those analyzed by Orduña et al. (2013). Initial link $-2=$ lever presses made to the lever associated with terminal links (TL) with a $2 \mathrm{~s}$ delay; Initial link $-28=$ lever presses made to the lever associated with terminal links with a $28 \mathrm{~s}$ delay; $(a, b)=$ parameters within the exponential function $a * \exp (b \mathrm{x})$; switch bias $=$ the number of switches in one direction/the number of switches in the opposite direction; return bias = the number of returns to the short-delay lever after a feeder entry/the number of returns to the long-delay lever after a feeder entry; $\mathrm{DUR}=$ duration; $\mathrm{CV}=$ coefficient of variation; $\mathrm{RPDE}$ $=$ recurrence period density entropy; $\mathrm{ACD}=$ autocorrelation difference, a measure of the difference between the measured autocorrelation function and an autocorrelation function for an aperiodic time series containing the same intervals

organization (see Fig. 5). This analysis of spatial structure in SOMs provided a way to compare the variability of response patterns across individuals, components, and sessions, and to determine whether specific behavioral measures such as response rate varied systematically as a function of experimental conditions.

Subjective analyses of component planes can reveal which behavioral measures were influenced by conditioning, but are less useful for identifying how these measures are related to experimental conditions or how they varied across individual rats. To provide a more objective assessment of SOM structure that would enable quantitative comparisons of structure across component planes, custom Matlab scripts were created that quantified structural properties of SOMs for each rat as a function of classes of inputs. The program first created a $4 \times 4 \mathrm{SOM}$ based on the full input set for a particular rat using MathWorks Neural Network Toolbox function selforgmap; the default neighborhood function and learning rate algorithm were used to train all SOMs. Next, the program partitioned inputs into three subsets consisting of two categories each: early or late components within a session; early or late sessions; and components associated with smaller or larger rewards. These partitioned input sets were used to calculate SOM Discrimination Indices for each behavioral measure. The program was run 100 times (creating 100 independent SOMs), and the mean SOM Discrimination Index values across these 100 simulations were used to rank each behavioral measure in terms of how strongly it determined the spatial organization of trained maps (i.e., identifying which behavioral measures varied most systematically for each rat within and across sessions and under which conditions).

Summary statistics for a subset of behavioral measures were also calculated and compared at the group level to further evaluate variability in behavioral descriptors within and across rats. Collectively, these analyses provided a way to evaluate the consistency of response patterns and their dynamics within and across sessions and individuals.

Analyzing condition-dependent variations Using analysis techniques identical to those described above for creating SOMs for individual rats, SOMs were also trained with an input set that included measures from all 12 rats. These maps made it possible to quantitatively assess differences in behavioral patterns between individual rats, as well as between different experimental conditions experienced by rats, including (1) comparisons between rats that experienced this particular reinforcement schedule (2-28) early in the experiment versus late in the experiment, (2) comparisons between components in which rats received either larger or smaller numbers of reinforcers; and (3) comparisons between rats that were reinforced using an FI schedule during the terminal link versus an FT schedule. Additionally, analyses of spatial organization within SOMs trained with data from all rats made it possible to evaluate whether the same systematic variations evident in the SOMs from individual rats were also evident in SOMs created from multiple rats. 
a

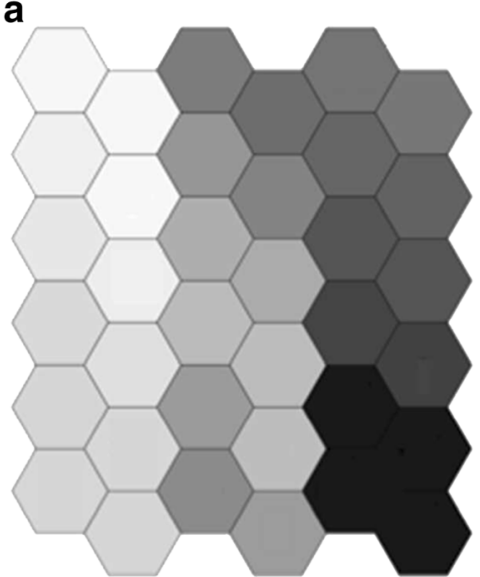

b

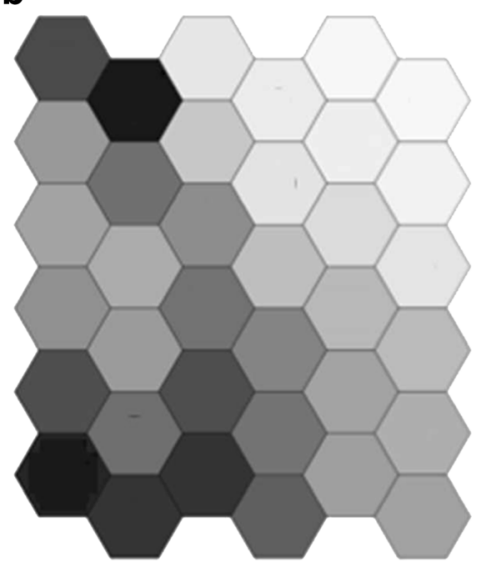

C

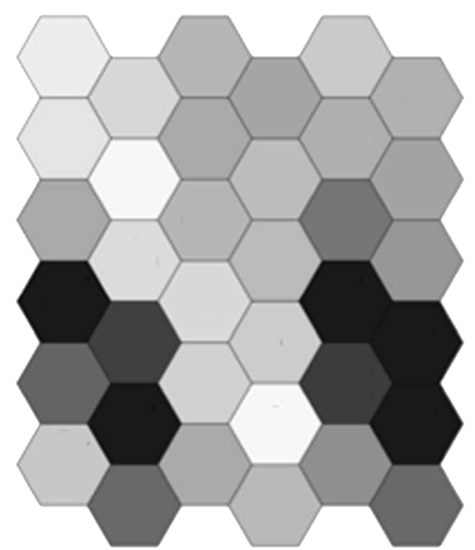

Fig. 5 Component planes from trained self-organizing maps (SOMs) show spatially organized sensitivities for behavioral measures that vary systematically within an input set. Each node within a $6 \times 6$ map is represented by a hexagon. The shading of each hexagon denotes the value of the behavioral measure that most strongly activates that node (darker shades indicate higher values). (a) Nodes in this component plan reveal a continuum of changes in shading from left to right, indicating that the spatial organization of this SOM is correlated with systematic variations in this behavioral measure (low values activate nodes on the

\section{Results}

\section{Matching behavior in the concurrent schedule}

To provide context for the current results, a brief overview of Orduña et al.'s (2013) results are given. In their study, the main dependent variable was responses per minute within initial links. Response rates varied as a function of delay of reinforcement in the terminal link, and sensitivity to delay was larger in components associated with a larger reinforcer. The reinforcement schedule in the terminal links (FT or FI) did not exert an influence. It was also found that within each component, preference developed very quickly, as evidenced by greater sensitivity to delay in the second halves of the components. Overall, the results suggest relatively direct relationships between reinforcement delay and response rate that are modulated by reward magnitude, but not by response contingencies within the terminal links.

\section{Regularities in response patterns}

Histograms of IRTs generally showed high densities of short intervals between responses (either lever presses or feeder entries) and more sparse long intervals. RPDE measures of lever pressing varied between 0.22 and 0.93 for individual responses across rats $(M d n \mathrm{~s}=0.41,0.33$, and 0.50 , for feeder entries, presses to the short-delay lever, and presses to the long-delay lever, respectively), suggesting an intermediate level of periodicity. Figure 6 shows that RPDE values for lever pressing at the group-level varied consistently both within and across sessions. Regularity of lever pressing at the group-level did not depend on whether rats were receiving larger or smaller rewards (Fig. 6c). left, higher values activate nodes on the right; see also Fig. 3b-d); this component plane would be classified as showing clear spatial organization. (b) A component plane that shows some spatial organization, with higher values in the lower left corner and lower values in the upper right corner. However, there are some nodes near the top that respond to higher values, and sensitivities to values are less systematically related to the diagonal spatial organization. (c) Nodes in this component plane show no clear spatial organization, with nodes responding to intermediate values spread throughout

Variations in RPDE measures of feeder entries were comparable to those for responses to the long-delay lever. RPDE measures from response bout time series were substantially higher than those associated with individual responses (range $=.55-.85$; $M d n=.71$ ), suggesting that bouts were less periodic.

\section{Individual variations in response patterns}

The systematic changes in RPDE measures shown in Fig. 6 predict that these measures should contribute to the spatial organization of SOMs. However, these group-level measures do not clearly reveal whether measures of temporal regularity would be more or less sensitive to experimental conditions than other measures such as response rates, or whether most individuals showed comparable trajectories of change. Analyses of SOMs trained with measures from individual rats provided a way to clarify these issues.

Visually coded classifications of component planes from the $6 \times 6$ SOMs for each rat revealed that the spatial structure of SOMs from most rats was mainly determined by (1) systematic variations in measures of IRT distributions for lever presses; (2) rate and regularity of pressing on the short-delay lever; and (3) rate and number of bouts on the long-delay lever. The behavioral measures that were least likely to impact the spatial organization of SOMs were variability of bout durations, biases in switching (which were typically miniscule), and periodicity of bout durations on the long-delay lever. The regularity of responding associated with each response option thus varied in ways that could not be predicted from overall response rates within initial links, and that were somewhat consistent across individuals. 

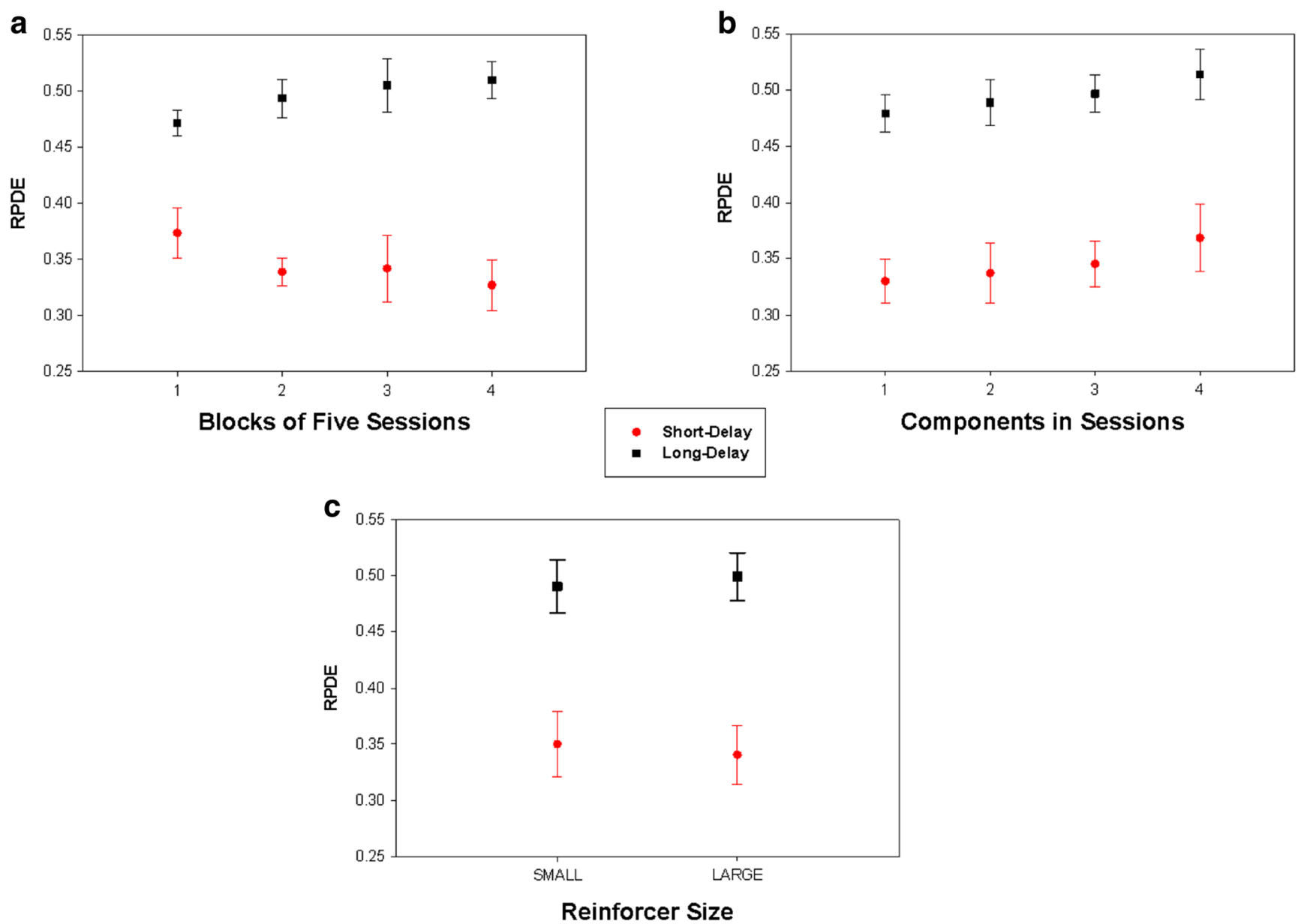

Fig. 6 Group-level means of recurrence period density entropy (RPDE) as a function of (a) session and (b) components within a session show systematic variations in response regularity. Lower RPDE values indicate

For 9 of 12 rats, differences in reinforcer magnitude were associated with the clearest spatial organization within SOMs (as determined through comparisons of SOM Discrimination Indices), with stage of training and time within a session leading to less structure. This finding indicates that changes in reinforcer magnitude led to systematic changes in response patterns for most rats. The behavioral measures that varied most consistently as a function of reinforcer magnitude differed across rats, but some response patterns were more likely to be affected than others including: number of bouts on the long-delay lever, IRT distribution for feeder entries, tendency to visit the feeder while pressing the short-delay lever, regularity of entering the feeder and of pressing the short-delay lever, and rate of responding (see Table 2). Note that most of these behavioral measures are not ones that would traditionally be analyzed in studies of two-components concurrentchains schedule tasks (i.e., dependent variables that are more sensitive to experimental conditions than response rates often are not being monitored). The SOMs for rats with little reinforcer-magnitude-related structure showed either spatial organization related to early and late sessions $(n=2)$, or to greater periodicity in response patterns. (c) RPDE shows no systematic variation as a function of reinforcer size. Error bars denote standard error of the mean

early or late components within sessions $(n=1)$, indicating that patterns in their responses were more strongly affected by the extent of training and time on task, respectively. Overall, 8 of 12 rats showed greater spatial organization related to early/ late sessions than for early/late components, suggesting that rats' responses varied more consistently across sessions than within sessions.

Figure 7 shows that some rats responded more regularly than others; no rats showed consistent evidence of either highly periodic responding or random responding.

An SOM created using the full data set from all 12 rats showed that individuals also varied significantly in terms of the range of behavioral responses that they exhibited within the operant chamber. There were consistencies in the response patterns produced by subsets of rats, as indicated by the fact that many SOM nodes within this map (17 of 36) responded to behavioral patterns produced by seven or more rats. However, other response patterns were idiosyncratic in that they were only observed in a small number of rats; two SOM nodes responded only to response patterns from a single rat and nine nodes responded only to patterns from three or four rats. 
Table 2 Rankings of the relative contribution of each behavioral measure to the spatial organization of self-organizing maps as determined through SOM Discrimination Indices

\begin{tabular}{|c|c|c|}
\hline Rank & $\operatorname{SOM}_{\text {Individuals }}(n=9$ rats $)$ & $\operatorname{SOM}_{\text {Group }}(n=12$ rats $)$ \\
\hline 1 & Number of bouts -28 & Switch bias \\
\hline 2 & Feeder entry $(a)$ & Feeder entry $(a)$ \\
\hline 3 & Return bias & Feeder entry \\
\hline 4 & RPDE Initial link -2 & Feeder entry $(b)$ \\
\hline 5 & Feeder entry $(b)$ & Initial link -2 $(a)$ \\
\hline 6 & RPDE Feeder entries & All responses $(a)$ \\
\hline 7 & Feeder entry & Initial link + TL -2 \\
\hline 8 & Initial link -2 & CV bout DUR -28 \\
\hline 9 & Initial link -28 & Initial link -2 (b) \\
\hline 10 & Initial link + TL -2 & Initial link -2 \\
\hline 11 & CV bout DUR - 28 & All responses $(b)$ \\
\hline 12 & Initial link -2 $(a)$ & Number of bouts -2 \\
\hline 13 & CV bout DUR -2 & Initial link -28 (b) \\
\hline 14 & Initial link + TL -28 & Return bias \\
\hline 15 & All responses $(b)$ & Number of bouts -28 \\
\hline 16 & Number of bouts - 2 & ACD bouts -28 \\
\hline 17 & Initial link -28 (a) & Number of switches \\
\hline 18 & Median bout DUR -2 & Initial link + TL -28 \\
\hline 19 & All responses $(a)$ & RPDE Initial link -28 \\
\hline 20 & ACD bouts -2 & Initial link -28 (a) \\
\hline 21 & Number of switches & Median bout DUR -2 \\
\hline 22 & Initial link -2 (b) & Initial link -28 \\
\hline 23 & ACD bouts -28 & CV bout DUR -2 \\
\hline 24 & Median bout DUR - 28 & RPDE Feeder entries \\
\hline 25 & Initial link -28 (b) & Median bout DUR -28 \\
\hline 26 & Switch bias & RPDE Initial link -2 \\
\hline 27 & RPDE Initial link - 28 & RPDE response bouts \\
\hline 28 & RPDE response bouts & ACD bouts -2 \\
\hline
\end{tabular}

Note: Italicized measures correspond to those analyzed by Orduña et al. (2013). Initial link $-2=$ lever presses made to the lever associated with terminal links (TL) with a $2 \mathrm{~s}$ delay; Initial link $-28=$ lever presses made to the lever associated with terminal links with a $28 \mathrm{~s}$ delay; $(a, b)=$ parameters within the exponential function $a * \exp (b x)$; switch bias $=$ the number of switches in one direction/the number of switches in the opposite direction; return bias = the number of returns to the short-delay lever after a feeder entry/the number of returns to the long-delay lever after a feeder entry; DUR = duration; $\mathrm{CV}=$ coefficient of variation; RPDE $=$ recurrence period density entropy; $\mathrm{ACD}=$ autocorrelation difference, $\mathrm{a}$ measure of the difference between the measured autocorrelation function and an autocorrelation function for an aperiodic time series containing the same intervals; $\mathrm{SOM}_{\text {Individuals }}$ rankings were based on SOM Discrimination Indices calculated from SOMs trained with data from individual rats reflecting how strongly each measure discriminated behavioral patterns associated with smaller or larger reinforcers; $\mathrm{SOM}_{\text {Group }}$ rankings were based on SOM Discrimination Indices calculated from SOMs trained with data from all rats, again reflecting how strongly a measure differentiated patterns associated with smaller or larger reinforcers.

Additionally, some rats produced a wide variety of response patterns during training (activating nodes spread throughout the SOM), whereas others responded much more stereotypically (activating few SOM nodes tightly clustered within one region).

Based on analyses of SOMs trained with data from individual rats, which showed clear spatial organization related to reinforcer magnitude for 9 out of 12 rats, one might expect that the spatial organization of an SOM trained with data from all 12 rats would also show spatial organization that was related to reinforcer magnitude. However, this was not the case. There was no obvious spatial organization related to reinforcer magnitude in SOMs trained with data from all rats (see Fig. 8a). In other words, an experimental condition that clearly led to differences in response patterns at the level of the individual, for most rats, showed no systematic effects at the group level. The factor of whether rats experienced the condition analyzed in this study either during their first exposure to concurrent schedules or after experiencing two similar schedules also showed no evidence of affecting the spatial organization of maps (see Fig. 8b), suggesting that extended experience performing the task under different reinforcement contingencies also does not lead to systematic behavioral changes at the group level. In contrast, the reinforcement schedule used during terminal links (either FI or FT) was strongly related to the spatial structure of SOMs trained with data from all 12 rats (see Fig. 8c), indicating that this experimental condition was systematically affecting how most rats responded in the task.

Analyses of component planes from the SOM trained with data from all 12 rats revealed that different terminal link reinforcement schedules led to systematic differences in IRT distributions and response rates for both levers, the number of bouts on the long-delay lever, and the number of switches between levers. Subjective visual analyses of component planes of more detailed $6 \times 6$ SOMs similarly showed that response rates, number of bouts, and IRT distributions on the short-delay lever, as well as the number of switches between levers, varied most systematically as a function of terminal link schedule at the group level. Sessions with FI schedules in terminal links were associated with faster rates of responding on both levers (producing shorter IRTs), and a greater numbers of switches between levers (leading to more bouts) relative to sessions with FT schedules in terminal links. Orduña et al. (2013) found no statistically significant effect of terminal link schedules on response rates within initial links when they analyzed performance in the last five sessions of training, suggesting that the effects of terminal link schedules on responding may be less evident later in training.

\section{Discussion}

What is seen in an animal's behavior depends in part on what the observer is looking for (Delprato, 1986; Iversen, 1991). Many past studies of operant conditioning have emphasized 


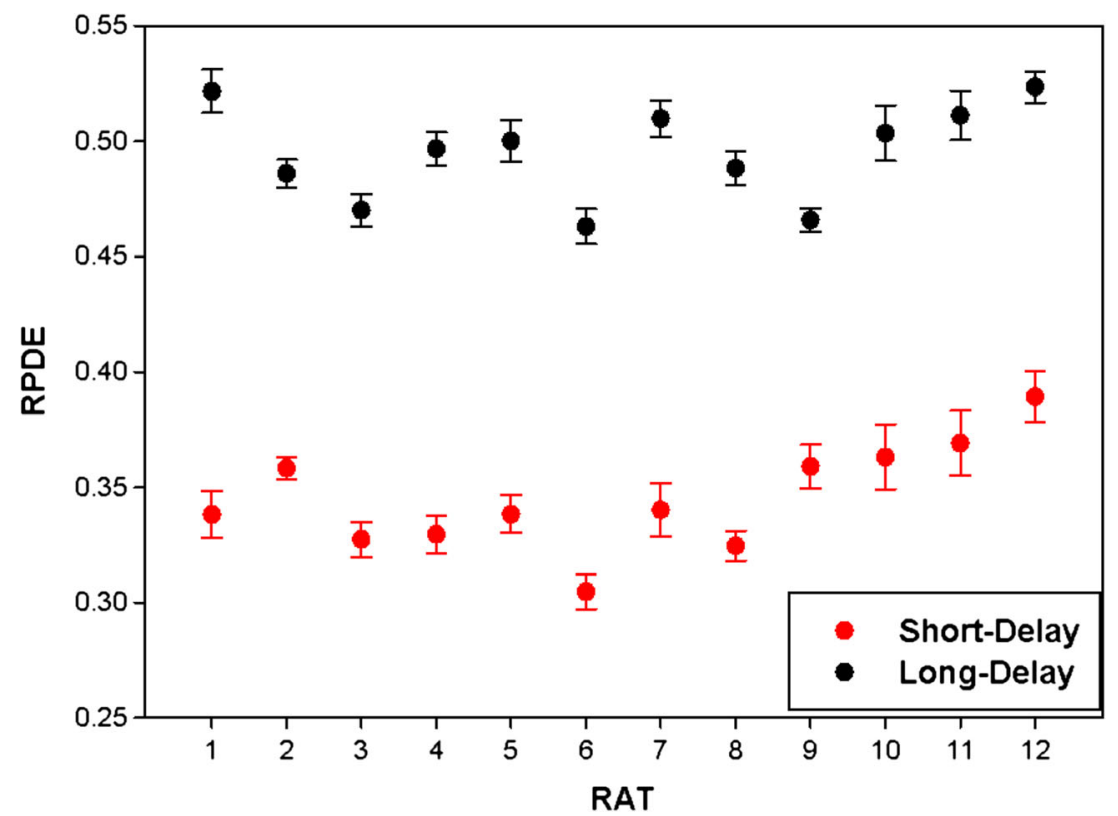

Fig. 7 Mean RPDE values for short-delay lever presses and long-delay lever presses for individual rats. Error bars denote standard error of the mean

stable "habits" induced by structured reinforcement schedules and on identifying response measures (e.g., absolute or relative response rate) that provide the clearest index of steadystate habit strength (Staddon \& Cerutti, 2003). The form and dynamics of habitual responses have generally been given less attention. In the case of concurrent-chain schedules, molar response rate measures are commonly used to develop and test various theories of how animals make choices. Some evidence suggests, however, that measures of responding that account for temporal patterning may provide more informative measures of behavior in such tasks (e.g., Shull et al., 2001). A major purpose of the current study was to evaluate whether more detailed measures of temporal regularities in responses produced within such choice tasks might reveal dynamic features of behavior during learning that are not captured by response rate measures.

A secondary goal of the study was to evaluate the efficacy of automated data analysis methods for describing trainingrelated variations in individual rat's response patterns over time. Guthrie and Horton's (1946) use of photographs and movies to record cats' behavior in puzzle boxes was a major methodological advance in terms of identifying regularities in movements across trials. Their analyses of these detailed records were limited to verbal descriptions of overall subjective a

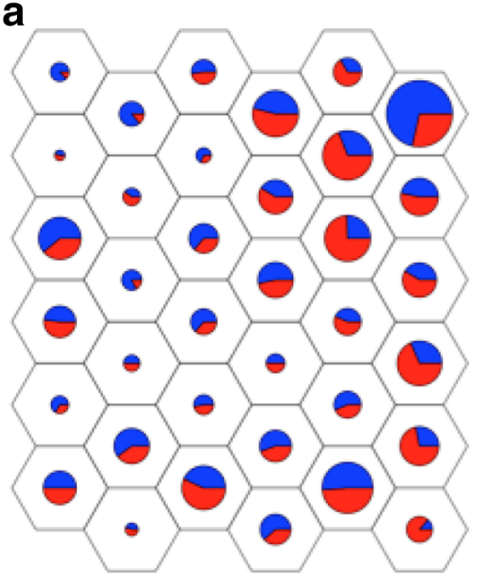

b

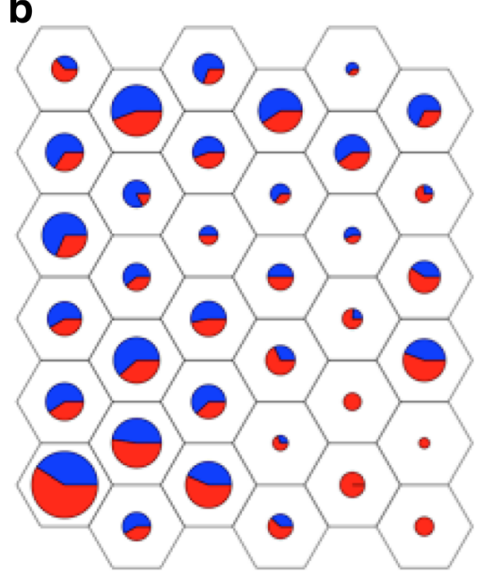

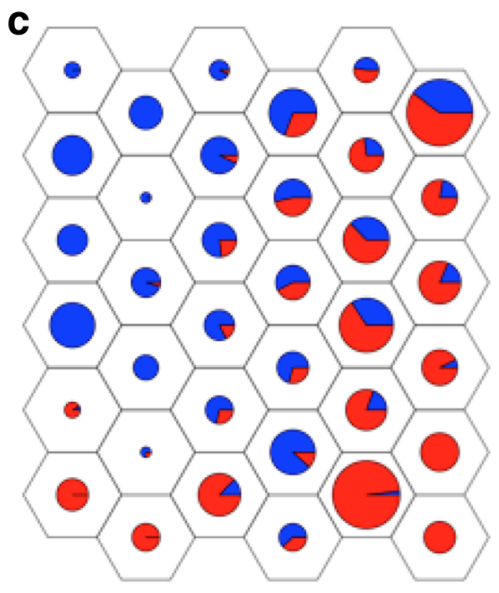

Fig. 8 (a) A self-organizing map (SOM) showing the distribution of activation for response patterns from components involving larger (blue/ dark gray) or smaller (red/light gray) reinforcement reveals limited spatial segregation associated with these 2 categories. (b) An SOM showing activations from patterns produced early in the experiment (blue/dark gray) versus later in the experiment (red/light gray) also reveals minimal spatial structure. (c) Activation associated with fixed interval terminal link schedules (blue/dark gray) or fixed time schedules (red/light gray) shows clear diagonal spatial organization. Maps were trained using all 28 behavioral measures collected from all 12 rats. Circle size denotes the number of patterns that best matched each SOM node 
impressions, however, because they could not quantify the images they collected in ways that would allow for objective assessments of variations in responding either within or across individuals. The current approach addressed this limitation by transforming behavioral records into time series and by using automated clustering techniques to sort descriptors of response patterns collected from these time series. This method can be viewed as a computational instantiation of the observational approach that Guthrie and Horton used in subjectively analyzing the learned movements of cats, but applied to rats' responses in an operant chamber rather than cats' responses in a puzzle box.

These two goals were pursued in an attempt to answer one basic question. What is the nature of the behavioral changes that occur as rats learn to make choices within a free-operant task? The current study revealed that the regularity of rats' responses under a multiple concurrent-chains schedule varied across conditions, individuals, and time. These findings raise questions about the adequacy of theoretical models of operant conditioning that do not account for such variations and that sometimes explicitly assume that such regularities do not exist. The study also showed that individual variations in rats' response patterns possessed idiosyncratic features that appear to be inconsistent with the assumption of uniformity of learning mechanisms across individuals (or any simple continuum of response variability across individuals). Finally, our findings suggest that although molar measures at the group level are generally consistent with measures of response patterns produced by individuals, the effects of varying conditions on responding by individuals are not sufficiently characterized by analyses of behavioral measures that are collapsed across groups of individuals.

\section{Relevance to understanding matching behavior}

Our results provide new perspective on past studies of matching behavior in choice experiments. In particular, several different response profiles can give rise to behavioral patterns that appear to be homogeneous when molar measures of responding are used. Consequently, measures of response rate at the group-level, or even for individuals, can give the false impression that identical reinforcement contingencies are inducing similar behavior patterns. A recent analysis of response patterns by monkeys performing in a two-arm bandit choice task reached similar conclusions (Laquitaine, Piron, Abellanas, Loewenstein, \& Boraud, 2013). In that study, researchers found that although the overall pattern of responding averaged across sessions was consistent with monkeys learning to respond preferentially to a more highly rewarded cue (probability matching), the performance of monkeys within individual sessions varied considerably across days. Even for a single monkey, the average learning curve across sessions was not representative of that monkey's response patterns within individual sessions. In the current study, rats showed consistent preferences for levers that generated terminal links with short delays within and across sessions. Nevertheless, the patterns of responding produced by individuals could vary considerably both within and across sessions in terms of both response rate and temporal regularity. Contrary to the assumption of several current theories of response selection that responses produced in choice tasks are probabilistic, the timing and periodicity of lever presses (and feeder entries) by individual rats in the current study changed systematically and predictably within and across sessions.

Although no existing models of choice behavior, or of operant conditioning more generally, are sufficiently detailed to make specific predictions about how rats' response timing should vary as a function of experience with complex choice tasks such as the one used in the current study, models that take into account the timing of individual responses can potentially provide insights into the possible sources of the observed patterns of responding (Cerutti \& Staddon, 2004; Cleaveland, 1999; Misak \& Cleaveland, 2011; Shimp, 1969, 1981). For example, Staddon's linear waiting model of responding in choice tasks proposes that the interval between recent reinforcement deliveries can strongly modulate the timing of an individual's responses (Staddon, Chelaru, \& Higa, 2002; Wynne, Staddon, \& Delius, 1996). Given that rats' lever presses help to determine when reinforcers are delivered, it seems likely that response timing might interact with timing of reward delivery to produce systematic fluctuations in the regularity of responding. Tanno and Silberberg (2012) suggested that the timing (and form) of responses might also reflect recently experienced patterns of responding (in particular, recent IRTs), such that ongoing patterns of responding might recapitulate subsets of recently performed temporal patterns of responding. Models such as these provide useful guides for how molecular measures of response regularity might be incorporated into theories of behavioral regulation that more accurately capture the systematic changes in individuals' actions during conditioning.

Temporal regularity in lever pressing during task performance varied systematically within and across sessions depending on whether the lever being pressed led to terminal links associated with short or long delays to rewards as well as on whether the rewards delivered were small or large in magnitude. In particular, regularity was greatest when rats were pressing a lever that led to quick, large rewards, a trend that increased over time both within and across sessions. These changes in response regularity occurred in parallel with increases in the overall rate of lever pressing, suggesting that faster pressing was typically more regular. Possibly, as rats increased their pressing rate, IRTs approached some asymptotic value, such that the increase in regularity reflected an increased number of IRTs at this duration. However, analyses of recurrence period density functions showed that highly 
regular response patterns did not generally show recurrence at a single frequency.

In many past studies of choice (including the experiment by Orduña et al., 2013), subjects have been trained for several sessions until they achieve stable patterns of responding that are consistent with the predictions of Herrnstein's matching law (Davison \& McCarthy, 1988; Williams, 1988). In other studies of choice, however, subjects seem to rapidly adapt their responses to match the differential reward outcomes associated with those responses (Berg \& Grace, 2006; Gallistel et al., 2007; Grace, Berg, \& Kyonka, 2006). If animals can rapidly adjust to changes in the outcomes of their responses, then why don't these adjustments show more stability across sessions? Our analyses suggest that factors other than differential reinforcement contribute to fluctuations in response patterns over both shorter (within session) and longer (across session) time scales. In particular, these analyses showed that variations in temporal regularity can themselves be periodic within the experimental context.

The behavioral response patterns of most rats were strongly affected by the magnitude of reinforcement. However, when SOMs were trained with behavioral descriptors from all 12 rats, and when summary statistics were compared at the group level, there was little evidence that systematic differences in responding were associated with differences in reward magnitude. Additionally, the effects of reward magnitude on rates of responding in the last five sessions appear to be relatively small (Orduña et al., 2013). These findings suggest that grouplevel measures may obscure the actual effects of differences in overall reward amount on behavioral patterns exhibited by rats in this concurrent choice task. Because the amount of reward associated with each lever is equal, it is unlikely that variations in lever preference are a major source of the observed differences (although the effect of reward magnitude on preference for immediacy indicates this could be a factor). Given that these response variations were less evident in SOMs trained with data from all rats, it is possible that the specific effects that larger or smaller rewards have on a rat's behavioral patterns vary across individuals. Consistent with this possibility, the behavioral measures that led to strong spatial separation of inputs associated with reward magnitude varied greatly across individual rats. As a result, it is not possible to reliably predict the effects of reward on behavioral patterns in this two choice task without knowing more details about the identity and behavioral profile of the rat for which predictions are being made.

A surprising discovery from these analyses was that the behavioral patterns produced by rats were strongly and consistently influenced by whether rewards were delivered on a FT schedule in the terminal link versus a FI interval. This effect was apparent regardless of the magnitude of reward and despite the fact that on average there was no significant difference in the delay of rewards for these two conditions after the onset of the terminal link. The main difference between these two types of terminal links was whether reward delivery was contingent on lever presses during the terminal link. Earlier work suggested that subjects should be indifferent to such variations in contingencies (Ferster, 1953; Ferster \& Hammer, 1965; Grace et al., 2006; Neuringer, 1969). Rats repeatedly pressed the lever during terminal links with FT schedules, so it is unclear whether rats on this schedule would be able to learn that rewards were not contingent on lever presses during the terminal link. Nevertheless, rats did typically respond differentially within initial links for the two types of terminal link schedules (generally pressing levers and switching between levers more often when terminal links involved an FI schedule), and rats also responded differentially during the terminal link depending on which schedule was in place (unpublished data). The fact that rats showed different patterns of responding in initial links, after receiving ostensibly equivalent rewards within terminal links suggests that response patterns during the terminal link in some way modulate response patterns during initial links, despite the fact that properties of terminal links (other than their onset) were not contingent on the response patterns produced during initial links. Interestingly, differences in responding associated with FT versus FI terminal link schedules were not limited to only variations in IRTs between lever presses or response rates, but also included an increased tendency for the rats to intersperse feeder entries among presses of the short-delay lever in initial links, and to switch between levers, suggesting that variations in terminal link schedules might have affected rats' responding more generally (e.g., by increasing or decreasing uncertainty about the predictability or contingency of outcomes).

\section{When is mapping of time series analyses of behavior warranted?}

Several of the analytical approaches used in the current study have never previously been used to measure behavior in any context. Consequently, it is reasonable to question whether these measures are suitable for characterizing response regularities in operant conditioning tasks. Most of the measures of temporal regularity included in the current study have previously been usefully applied to the analysis of time series of biological signals. Additionally, SOMs have been used in numerous fields to sort a wide range of inputs. Thus, the analysis techniques themselves are well-validated methods for characterizing and classifying natural phenomena. In a few cases, SOMs have been used to describe the movement patterns of humans (Barton, 2006; Perl \& Dauscher, 2006) and robots (Wünstel, Polani, Uthmann, \& Perl, 2001). Ultimately, the utility of these techniques for describing and understanding variability or stereotypy in animal behavior can only be evaluated by applying the techniques and observing the outcomes. 
The power of these quantitative approaches for describing behavior dynamics is limited primarily by the precision and quality of the behavioral measures that are collected.

Neural networks have rarely been used to analyze the results of conditioning experiments, partly because of emphases on molar measures of responding such as response rate. When response properties can be summarized with a single number, there is little value in applying a neural network to analyze how this number varies. However, in cases where multiple measures of behavior must be simultaneously considered, neural networks provide a powerful tool for objectively identifying which measures are most informative (Kohonen, 2013; Lloyd et al., 2009; Mercado, 2011). One goal of the current analyses was to evaluate whether behavioral measures at different time scales would provide useful information about learning-related changes in responses beyond what measures of response rates revealed. Toward that goal we evaluated variations in 28 separate behavioral measures, collected from 12 rats, across 80 sequential components per rat. Although standard descriptive statistical techniques could in principle be used to analyze variations in these measures (e.g., through plots of mean values, factor analyses, multidimensional scaling), most of these techniques assume linear relationships between variables and uniformity in the sources of variance across time and subjects. These assumptions are unlikely to hold true for response patterns produced by rats in an operant conditioning task (Mercado, 2011; Tryon, 1940). Results from SOM analyses confirmed that many of the response variations occurring within a multiple concurrent-chains schedule task would be either obscured or distorted by traditional statistical approaches. Such distortions are a result of idiosyncratic behavioral profiles shown by individual rats.

Pavlov (1927) suggested early on that intrinsic variations between individuals could have profound effects on how they learned as well as what they learned. He focused primarily on the effects such individual variations had on the methods through which adequate conditioning could be achieved as well as specific accommodations in training that could be made to counteract difficulties associated with noncooperative subjects. More recently, researchers have emphasized differences in the relative ease and speed with which subgroups of individuals learn particular tasks when no such accommodations are made, for example, by correlating faster learning with particular behaviorally identified traits, such as "boldness" (Dugatkin \& Alfieri, 2003; Guillette, Hahn, Hoeschele, Przyslupski, \& Sturdy, 2014). There do not appear to be any prior studies that have investigated whether individuals vary systematically in terms of how regular/stereotypical their behavior is during the learning and performance of conditioning tasks, although it is well known that species and individuals can vary considerably in their tendency to perseverate in performing particular (often undesired) responses during the acquisition of such tasks (e.g., Orduña, ValenciaTorres, \& Bouzas, 2009).

Details regarding behavioral stereotypy can be important for evaluating the adequacy and predictive utility of competing theories of conditioning (MacDonall, 2009; Tanno \& Silberberg, 2012), as well as for assessing the plausibility of basic assumptions that underlie traditional theories of learning and behavior. The stability of automatically extracted behavioral measures was evaluated within and across rats using automated clustering by SOMs, thereby reducing the potential for post hoc subjective or selective biases to influence measurements or interpretations of temporally dynamic changes in behavior. The value of this approach is shown in part by several regularities in behavioral responses revealed by SOM analyses that have not been previously noted in studies of choice behavior. For instance, studies of choice have often focused on measuring the rate at which subjects press levers (or peck keys) for food reinforcement under various reinforcement schedules. Ranking behavioral measures based on how systematically they varied as a function of experimental conditions revealed that rates of feeder entries were actually more sensitive to experimental conditions than rates of lever pressing in a two-component multiple concurrent-chains schedule task. The SOM analyses also showed that the behavioral measures that varied with experimental conditions at the group level only partially overlapped with the measures that were varying in individual rats.

Past analyses of stereotypical responding in operant tasks have consistently noted that the recurrent patterns produced within such tasks are often idiosyncratic to the individuals producing them. Guthrie and Horton (1946) noted that the particular patterns of individual cats' responses were so characteristic that it was possible to sort traces of response postures from different individuals based on their unique features. In the current study, a subset of rats produced temporal response patterns that were so distinctive that SOMs trained with data from all 12 rats could be used to identify the specific rat that produced some patterns. In other cases, particular patterns of responding were produced by only a small subset of rats. Similarly, although most rats showed differential response patterns based on reward magnitude, three rats ( $25 \%$ of those tested) did not. The regularity of responding also varied across rats, as did variations in response regularity over time. Although all rats showed uniformity with respect to certain response patterns (e.g., unbiased switching between levers, modulating response rates relative to the immediacy of reward), the overall response profiles of rats were more indicative of heterogeneity in response patterning than homogeneity. This heterogeneity was not simply a consequence of randomly timed response production, or differences in the variable intervals at which reinforcers were delivered, because the patterns often changed systematically within and across sessions. Instead, like the 
actions of cats in a puzzle box, variability (and consistency) in responding within and between rats likely reflected both variations in individual propensities and relatively subtle variations in the rats' experiences within the chamber. SOMs trained with measures of response regularity provide a novel index of behavioral variability that can be used to study animal learning in both natural and laboratory settings.

\section{Summary and conclusions}

Molar and molecular measures of responding were analyzed simultaneously (as recently advocated by Shimp, 2014), making it possible to directly compare the adequacy of each type of measure for describing patterns of behavior at both the individual and group level. SOM-based analyses of systematic variations in response regularity within a complex operant conditioning task suggest that both types of measures are important for tracking changes in behavior within and across conditions, time, and individual rats.

Several unsuspected features of rats' responses in a choice task were discovered, indicating that the analytical approach used here can provide unique scientific insights into learning phenomena. These findings include that (1) the regularity of response patterns varied as a function of number of training sessions, time on task, magnitude of reinforcement, and reinforcement contingencies; (2) individuals showed heterogeneous, stereotyped patterns of responding, despite similarities in matching behavior; (3) trajectories of behavioral variation within individuals were less evident in group-level analyses; and (4) reinforcement contingencies within terminal links that theoretically should have had little effect on responding strongly modulated response patterns. A critical step in developing theories of learning and behavior that can account for behavior dynamics within free operant tasks is to develop objective methods of detecting and tracking regularities in subjects' responses. Greater precision in behavioral descriptions and analyses allow for more thorough theoretical analyses and more specific predictions. Similarly, developing methods that more fully and adequately account for systematic differences between the behavior of individuals within conditioning tasks can potentially provide new insights into the mechanisms that determine learning-related variations in the behavior of actual, as opposed to hypothetical, individuals.

Author Note This research was supported by Grants IN305012 from PAPIIT and 167016 from CONACYT, by NSF grant \#SBE-0542013 to the Temporal Dynamics of Learning Center, an NSF Science of Learning Center, and by a Faculty Internationalization Fund award from the University at Buffalo, SUNY.

\section{References}

Barton, G. J. (2006). Visualisation of clinical gait data using a selforganising artificial neural network. In R. Begg \& M. Palaniswami (Eds.), Computational intelligence for movement sciences: Neural networks and other emerging techniques (pp. 197-216). Hershey, PA: Idea Group.

Baum, W. M. (2002). From molecular to molar: A paradigm shift in behavior analysis. Journal of the Experimental Analysis of Behavior, 78, 95-116. doi:10.1901/jeab.2002.78-95

Baum, W. M., \& Rachlin, H. C. (1969). Choice as time allocation. Journal of the Experimental Analysis of Behavior, 12, 861-874.

Berg, M. E., \& Grace, R. C. (2006). Initial-link duration and acquisition of preference in concurrent chains. Learning \& Behavior, 34, 50-60.

Blough, D. S. (1963). Interresponse time as a function of continuous variables: A new method and some data. Journal of the Experimental Analysis of Behavior, 6, 237-246.

Budaev. (2010). Using principal components and factor analysis in animal behaviour research: Caveats and guidelines. Ethology, 116, 472-480.

Cerutti, D. T., \& Staddon, J. E. (2004). Time and rate measures in choice transitions. Journal of the Experimental Analysis of Behavior, 81, 135-154. doi:10.1901/jeab.2004.81-135

Cleaveland, J. M. (1999). Interresponse-time sensitivity during discretetrial and free-operant concurrent variable-interval schedules. Journal of the Experimental Analysis of Behavior, 72, 317-339. doi:10.1901/jeab.1999.72-317

Davison, M., \& McCarthy, D. (1988). The matching law: A research review. Hillsdale, NJ: Erlbaum.

Delprato, D. J. (1986). Response patterns. In H. W. Reese \& L. J. Parrott (Eds.), Behavior science: Philosophical, methodological, and empirical advances (pp. 61-113). Hillsdale, NJ: Erlbaum.

Demsar, J., Zupan, B., Leban, G., \& Curk, T. (2004). Orange: From experimental machine learning to interactive data mining. Lecture Notes in Computer Science, 3202, 537-539.

Dugatkin, L. A., \& Alfieri, M. S. (2003). Boldness, behavioral inhibition and learning. Ethology Ecology \& Evolution, 15, 43-49.

Eckmann, J.-P., Oliffson Kamphorst, S., \& Ruelle, D. (1987). Recurrence plots of dynamical systems. Europhysics Letters, 4, 973-977.

Fantino, E., \& Royalty, P. (1987). A molecular analysis of choice on concurrent-chains schedules. Journal of the Experimental Analysis of Behavior, 48, 145-159. doi:10.1901/jeab.1987.48-145

Ferster, C. B. (1953). Sustained behavior under delayed reinforcement. Journal of Experimental Psychology, 45, 218-224.

Ferster, C. B., \& Hammer, C. (1965). Variables determining the effects of delay in reinforcement. Journal of the Experimental Analysis of Behavior, 8, 243-254.

Galbicka, G. (1992). The dynamics of behavior. Journal of the Experimental Analysis of Behavior, 57, 243-248.

Gallistel, C. R., Fairhurst, S., \& Balsam, P. (2004). The learning curve: Implications of a quantitative analysis. Proceedings of the National Academy of Sciences, 101, 13124-13131. doi:10.1073/pnas. 0404965101

Gallistel, C. R., King, A. P., Gottlieb, D., Balci, F., Papachristos, E. B., Szalecki, M., \& Carbone, K. S. (2007). Is matching innate? Journal of the Experimental Analysis of Behavior, 87, 161-199.

Gentry, G. D., Weiss, B., \& Laties, V. G. (1983). The microanalysis of fixed-interval responding. Journal of the Experimental Analysis of Behavior, 39, 327-343.

Grace, R. C., Berg, M. E., \& Kyonka, E. G. (2006). Choice and timing in concurrent chains: Effects of initial-link duration. Behavioral Processes, 71, 188-200. doi:10.1016/j.beproc.2005.11.002

Guillette, L. M., Hahn, A. H., Hoeschele, M., Przyslupski, A. M., \& Sturdy, C. B. (2014). Individual differences in learning speed, performance accuracy and exploratory behaviour in black-capped 
chickadees. Animal Cognition, 18, 165-178. doi:10.1007/s10071014-0787-3

Guthrie, E. R., \& Horton, G. P. (1946). Cats in a puzzle box. New York, NY: Rinehart \& Company.

Herrnstein, R. J. (1970). On the law of effect. Journal of the Experimental Analysis of Behavior, 13, 243-266.

Iversen, I. H. (1991). Methods of analyzing behavior patterns. In I. H. Iversen \& K. A. Lattal (Eds.), Experimental analysis of behavior (pp. 193-241). Amsterdam, The Netherlands: Elsevier.

Kirkpatrick, K., \& Church, R. M. (2003). Tracking of the expected time to reinforcement in temporal conditioning procedures. Learning \& Behavior, 31, 3-21.

Kirt, T., \& Vainik, E. (2007). Comparison of the methods of selforganizing maps and multidimensional scaling in analysis of Estonian emotion concepts. In J. Nivre, H.-J. Kaalep, K. Muiscnek, \& M. Koit (Eds.), Proceedings of the 16th Nordic conference on computational linguistics (pp. 113-120). Tartu, Estonia: University of Tartu.

Kohonen, T. (2001). Self-organizing maps. Berlin, Germany: Springer.

Kohonen, T. (2013). Essentials of the self-organizing map. Neural Networks, 37, 52-65. doi:10.1016/j.neunet.2012.09.018

Laquitaine, S., Piron, C., Abellanas, D., Loewenstein, Y., \& Boraud, T. (2013). Complex population response of dorsal putamen neurons predicts the ability to learn. PLoS One, 8, e80683. doi:10.1371/ journal.pone. 0080683

Li, J. S., \& Huston, J. P. (2002). Non-linear dynamics of operant behavior. Reviews in the Neurosciences, 13, 31-57.

Li, J. S., Krauth, J., \& Huston, J. P. (2006). Operant behavior of rats under fixed-interval reinforcement schedules: A dynamical analysis via the extended return map. Nonlinear Dynamics, Psychology, and Life Sciences, 10, 215-240.

Little, M. A., McSharry, P. E., Moroz, I. M., \& Roberts, S. J. (2006, May 14-19). Nonlinear, biophysically-informed speech pathology detection (pp. 1080-1083). IEEE International Conference on Acoustics, Speech and Signal Processing, ICASSP 2006, Toulouse, France.

Little, M. A., McSharry, P. E., Roberts, S. J., Costello, D. A. E., \& Moroz, I. M. (2007). Exploiting nonlinear recurrence and fractal scaling properties for voice disorder detection. Biomedical Engineering Online, 6, 23. doi:10.1186/1475-925X-6-23

Lloyd, G. R., Wongravee, K., Silwood, C. J. L., Grootveld, M., \& Brereton, R. G. (2009). Self organising maps for variable selection: Application to human saliva analysis by nuclear magnetic resonance spectroscopy to investigate the effect of an oral healthcare product. Chemometrics and Intelligent Laboratory Systems, 98, 149-161.

MacDonall, J. S. (2009). The stay/switch model of concurrent choice. Journal of the Experimental Analysis of Behavior, 91, 21-39.

Marr, M. J. (1992). Behavior dynamics: One perspective. Journal of the Experimental Analysis of Behavior, 57, 249-266.

Mercado, E., III. (2011). Mapping individual variations in learning capacity. International Journal of Comparative Psychology, 24, 4-35.

Misak, P., \& Cleaveland, J. M. (2011). Preference as a function of active interresponse times: A test of the active time model. Journal of the Experimental Analysis of Behavior, 96, 215-225. doi:10.1901/jeab. 2011.96-215

Neuringer, A. J. (1969). Delayed reinforcement versus reinforcement after a fixed interval. Journal of the Experimental Analysis of Behavior, 12, 375-383.

Nevin, J. A., \& Grace, R. C. (2000). Behavioral momentum and the law of effect. Behavioral and Brain Sciences, 23, 73-90.

Newland, M. C. (1997). Quantifying the molecular structure of behavior: Separate effects of caffeine, cocaine, and adenosine agonists on interresponse times and lever-press durations. Behavioral Pharmacology, 8, 1-16.

Orduña, V., Valencia-Torres, L., Cruz, G., \& Bouzas, A. (2013). Sensitivity to delay is affected by magnitude of reinforcement in rats. Behavioural Processes, 98, 18-24. doi:10.1016/j.beproc. 2013.04.011

Orduña, V., Valencia-Torres, L., \& Bouzas, A. (2009). DRL performance of spontaneously hypertensive rats: Dissociation of timing and inhibition of responses. Behavioural Brain Research, 201, 158-165. doi:10.1016/j.bbr.2009.02.016

Ossenkopp, K. P., Sorenson, L., \& Mazmanian, D. S. (1994). Factor analysis of open-field behavior in the rat (Rattus norvegicus): Application of the three-way PARAFAC model to a longitudinal data set. Behavioural Processes, 31, 129-144. doi:10.1016/03766357(94)90001-9

Palya, W. L. (1992). Dynamics in the fine structure of schedule-controlled behavior. Journal of the Experimental Analysis of Behavior, 57, 267-287. doi:10.1901/jeab.1992.57-267

Pavlov, I. P. (1927). Conditioned reflexes. London, England: Oxford University Press.

Pear, J. J. (1985). Spatiotemporal patterns of behavior produced by variable-interval schedules of reinforcement. Journal of the Experimental Analysis of Behavior, 44, 217-231.

Pear, J. J. (2001). The science of learning. Philadelphia, PA: Taylor \& Francis.

Perl, J., \& Dauscher, P. (2006). Dynamic pattern recognition in sport by means of artificial neural networks. In R. Begg \& M. Palaniswami (Eds.), Computational intelligence for movement sciences: Neural networks and other emerging techniques (pp. 299-319). Hershey, PA: Idea Group.

Rachlin, H. C. (2000). The science of self-control. Cambridge, MA: Harvard University Press.

Shimp, C. P. (1966). Probabilistically reinforced choice behavior in pigeons. Journal of the Experimental Analysis of Behavior, 9, 443455. doi:10.1901/jeab.1966.9-443

Shimp, C. P. (1969). The concurrent reinforcement of two interresponse times: The relative frequency of an interresponse time equals its relative harmonic length. Journal of the Experimental Analysis of Behavior, 12, 403-411.

Shimp, C. P. (1981). Local structure of steady-state operant behavior. In C. M. Bradshaw, E. Szabadi, \& C. F. Lowe (Eds.), Quantification of steady-state operant behaviour (pp. 189-203). Amsterdam: Elsevier.

Shimp, C. P. (1992). Computational behavior dynamics: An alternative description of Nevin (1969). Journal of the Experimental Analysis of Behavior, 57, 289-299. doi:10.1901/jeab.1992.57-289

Shimp, C. P. (2014). How molecular, molar and unified analyses change the meaning of behavioral variability. International Journal of Comparative Psychology, 27, 224-247.

Shimp, C. P., Fremouw, T., Ingebritsen, L. M., \& Long, K. A. (1994). Molar function depends on molecular structure of behavior. Journal of Experimental Psychology: Animal Behavior Processes, 20, 96 107.

Shull, R. L., Gaynor, S. T., \& Grimes, J. A. (2001). Response rate viewed as engagement bouts: Effects of relative reinforcement and schedule type. Journal of the Experimental Analysis of Behavior, 75, 247274. doi:10.1901/jeab.2001.75-247

Sidman, M. (1954). The temporal distribution of avoidance responses. Journal of Comparative and Physiological Psychology, 47, 399402.

Silva, F. J., \& Pear, J. J. (1995). Stereotypy of spatial movements during noncontingent and contingent reinforcement. Animal Learning and Behavior, 23, 245-255.

Silva, F. J., Pear, J. J., Tait, R. W., \& Forest, J. J. (1996). Fourier analysis of movement patterns in pigeons. Behavior Research Methods, Instruments, \& Computers, 28, 27-37.

Staddon, J. E., \& Cerutti, D. T. (2003). Operant conditioning. Annual Review of Psychology, 54, 115-144. doi:10.1146/annurev.psych. 54.101601 .145124 
Staddon, J. E., Chelaru, I. M., \& Higa, J. J. (2002). A tuned-trace theory of interval-timing dynamics. Journal of the Experimental Analysis of Behavior, 77, 105-124. doi:10.1901/jeab.2002.77-105

Tanno, T., \& Silberberg, A. (2012). The copyist model of response emission. Psychonomic Bulletin \& Review, 19, 759-778. doi:10.3758/ s13423-012-0267-1

Tryon, R. C. (1940). Studies of individual differences in maze ability: VII. The specific components of maze ability and a general theory of psychological components. Journal of Comparative Psychology, $30,283-335$

Webber, C. L., Jr., \& Zbilut, J. P. (1994). Dynamical assessment of physiological systems and states using recurrence plot strategies. Journal of Applied Physiology, 76, 965-973.

Weiss, B. (1970). The fine structure of operant behavior during transition states. In W. Schoenfeld (Ed.), The theory of reinforcement schedules (pp. 277-311). New York, NY: Appleton-Century-Crofts.

Williams, B. A. (1988). Reinforcement, choice, and response strength. In R. C. Atkinson, R. J. Herrnstein, G. Lindzey, \& R. D. Luce (Eds.),
Stevens handbook of experimental psychology (Vol. 2, pp. 167244). New York, NY: John Wiley \& Sons.

Williams, B. A. (1990). Enduring problems for molecular accounts of operant behavior. Journal of the Experimental Analysis of Behavior, 16, 213-216.

Worthy, D. A., Hawthorne, M. J., \& Otto, A. R. (2013). Heterogeneity of strategy use in the Iowa gambling task: A comparison of win-stay/ lose-shift and reinforcement learning models. Psychonomic Bulletin \& Review, 20, 364-371. doi:10.3758/s13423-012-0324-9

Wünstel, M., Polani, D., Uthmann, T., \& Perl, J. (2001). Behavior classification with self-organizing maps. In P. Stone, T. Balch, \& G. Kraetzschmar (Eds.), Robocup 2000, LNAI 2019 (pp. 108-118). Berlin, Germany: Springer-Verlag.

Wynne, C. D., Staddon, J. E., \& Delius, J. D. (1996). Dynamics of waiting in pigeons. Journal of the Experimental Analysis of Behavior, 65, 603-618. 